\title{
Radiologic-pathologic discordance in biopsy-proven usual interstitial pneumonia
}

\author{
Kunihiro Yagihashi $i^{1,2,3}$, Jason Huckleberry ${ }^{4}$, Thomas V. Colby ${ }^{5}$, \\ Henry D. Tazelaar ${ }^{5}$, Jordan Zach ${ }^{2}$, Baskaran Sundaram ${ }^{6}$, Sudhakar Pipavath ${ }^{7}$, \\ Marvin I. Schwarz ${ }^{8}$ and David A. Lynch ${ }^{2}$ for the Idiopathic Pulmonary Fibrosis \\ Clinical Research Network (IPFnet) ${ }^{9}$
}

Affiliations: 'Dept of Radiology, St Marianna University School of Medicine, Kanagawa, Japan. ${ }^{2}$ Dept of Radiology, National Jewish Health, Denver, CO, USA. ${ }^{3}$ Dept of Radiology, St Luke's International Hospital, Tokyo, Japan. ${ }^{4}$ Dept of Radiology, CPMG Kaiser Colorado, Denver, CO, USA. ${ }^{5}$ Dept of Laboratory Medicine and Pathology, Mayo Clinic, Phoenix, AZ, USA. 'Dept of Radiology, Thomas Jefferson University Hospital, Philadelphia, PA, USA. ${ }^{7}$ Dept of Radiology, University of Washington, Seattle, WA, USA. ${ }^{8}$ Dept of Medicine, University of Colorado Denver, School of Medicine, Aurora, CO, USA. ${ }^{9}$ Members of the Idiopathic Pulmonary Fibrosis Clinical Research Network are listed in the online supplementary material.

Correspondence: Kunihiro Yagihashi, Dept of Radiology, St Marianna University School of Medicine, 2-16-1 Sugao, Miyamae-ku, Kawasaki, Kanagawa, 216-8511, Japan. E-mail: k2yagihashiamarianna-u.ac.jp

ABSTRACT The aim of this study was to compare the clinical, radiological and histological findings in a large population of subjects enrolled during a multicentre study of idiopathic pulmonary fibrosis, with a focus on discordance between imaging and histologic diagnoses of usual interstitial pneumonia (UIP).

Two independent radiologists retrospectively reviewed 241 subjects who underwent high-resolution computed tomography (HRCT) and surgical lung biopsies. HRCT findings were classified as UIP, possible UIP and inconsistent with UIP. Histological findings were classified as definite, probable, possible and not UIP.

Of the 241 cases, 102 (42.3\%) had HRCT findings of UIP, 64 (26.6\%) had possible UIP and 75 (31.1\%) were inconsistent with UIP. Among those with UIP on HRCT, 99 (97.1\%) had histologically definite or probable UIP (concordant group), and 71 (94.7\%) of those with "inconsistent" HRCT features had histologically definite or probable UIP (discordant group). Discordant subjects were slightly younger and less likely to be smokers than concordant subjects, but no survival differences were identified.

In this population of patients enrolled with a diagnosis of idiopathic pulmonary fibrosis, $94.7 \%$ of those with HRCT findings "inconsistent with UIP" demonstrated histological UIP. This suggests that the term "inconsistent with UIP" is misleading.

@ERSpublications

Individuals with fibrosing interstitial pneumonia who have CT findings "inconsistent with UIP" may have IPF http://ow.ly/WaufM

For editorial comment see Eur Respir J 2016; 47: 1057-1059 [DOI: 10.1183/13993003.00265-2016].

This article has supplementary material available from erj.ersjournals.com

Received: Aug 142015 | Accepted after revision: Dec 042015 | First published online: Feb 252016

Conflict of interest: Disclosures can be found alongside the online version of this article at erj.ersjournals.com

Copyright OERS 2016 


\section{Introduction}

The idiopathic interstitial pneumonias (IIPs) are a diverse group of parenchymal lung diseases delineated in the American Thoracic Society (ATS)/European Respiratory Society (ERS) classification [1]. Idiopathic pulmonary fibrosis (IPF), the most common IIP, is a chronic progressive lung disease of unknown cause that is characterised by a histopathological and computed tomography (CT) pattern of usual interstitial pneumonia (UIP). Compared to other IIPs, IPF has a significantly worse prognosis, with a median survival duration as low as 2.1 years following diagnosis [2]. Therefore, it is important to distinguish IPF from other IIPs. High-resolution computed tomography (HRCT) is an essential component of the diagnostic algorithm for IPF. According to the ATS/ERS/Japanese Respiratory Society (JRS)/Latin American Thoracic Association (ALAT) guidelines published in 2011, the fibrosis observed on HRCT should be classified as UIP, possible UIP and inconsistent with UIP [3]. The characteristic HRCT features of UIP are a predominantly subpleural and basal distribution, reticular abnormalities and honeycombing.

In the appropriate clinical setting, the presence of a pattern suggestive of UIP on HRCT is sufficient for the diagnosis of IPF, obviating the need for a surgical lung biopsy (SLBx). However, biopsy-proven UIP is also seen in patients without typical HRCT features of UIP [1, 3, 4]. Patients with non-typical HRCT features of UIP may have a better prognosis than those with typical HRCT features of UIP [2, 5]. Furthermore, the clinical features and outcomes in those patients with radiological-pathological discordance are not well defined. The goals of the current study are to compare radiological and histological diagnoses in a large population of subjects enrolled in a multicentre study of IPF, and to compare clinical, radiological and histological findings, in addition to outcomes, in subjects with concordant and discordant radiological and pathological diagnoses.

\section{Methods}

\section{Patient selection}

We retrospectively reviewed the HRCT and histological diagnoses in a large population of subjects enrolled in three studies sponsored by the Idiopathic Pulmonary Fibrosis Clinical Research Network (IPFnet) research network at 26 sites throughout the USA (Prednisone, Azathioprine, and N-Acetylcysteine: a Study that Evaluates Response in Idiopathic Pulmonary Fibrosis (PANTHER-IPF), Sildenafil Trial of Exercise Performance in Idiopathic Pulmonary Fibrosis (STEP-IPF) and Anticoagulant Effectiveness in Idiopathic Pulmonary Fibrosis (ACE-IPF)) [6-8]. The studies were designed and conducted by the IPFnet steering committee and were performed at 26 clinical centres in the USA. The independent IPFnet protocol review committee, the IPFnet data and safety monitoring board and local institutional review boards approved the protocols. All patients provided written informed consent. Details regarding study enrolment are provided in the online supplementary material.

CT scans were available for 539 subjects (236 from PANTHER-IPF, 169 from STEP-IPF and 134 from ACE-IPF). Among these cases, 241 subjects (111 from PANTHER-IPF, 65 from STEP-IPF and 65 from ACE-IPF) had undergone SLBx, and these formed the study population. Data evaluated included age, sex, duration of illness, smoking history, history of antigen exposure, gastro-oesophageal reflux symptoms, post-walk Borg dyspnoea score, total score on the St George's Respiratory Questionnaire (SGRQ), the 6-min walk test (6MWT), pulmonary function tests (PFT), HRCT and SLBx findings.

\section{HRCT imaging technique and analysis}

HRCT scans were obtained at 26 centres using a variety of scanners between 2007 and 2012. All subjects had HRCT with between $0.5-$ and $1.25-\mathrm{mm}$ slice thickness at $1-\mathrm{cm}$ intervals, at least two expiratory scans (if there was inspiratory mosaic attenuation) and at least four prone scans (if there was any dependent density on the supine images).

The images were reviewed retrospectively in random order by two experienced thoracic radiologists (Kunihiro Yagihashi: 10 years of experience and Jason Huckleberry: 5 years of experience). The images were assessed independently and classified according to the ATS/ERS/JRS/ALAT guidelines into UIP, possible UIP and inconsistent with UIP patterns. The observers were blinded to the clinical information and histological diagnoses. Additional details regarding the radiological evaluations are provided in the online supplementary material.

\section{Pathological analysis}

SLBx were performed in 241 out of the 539 subjects. The samples were reviewed by the local and central pathologists and classified pathologically into definite, probable, possible and not UIP using the ATS/ERS/ JRS/ALAT guidelines [3]. Additional details of histological evaluation are provided in the online supplementary material. 


\section{Statistical analysis}

All statistical analysis was performed using JMP version 10 (SAS Institute Inc., Cary, NC, USA) statistical software. Comparisons between the baseline characteristics for continuous measurements were analysed using one-way ANOVA (or Kruskal-Wallis test if data were non-normal) for three group comparisons, which were followed by the Tukey-Kramer (or the Steel-Dwass test if the data were nonparametric) and t-test (or Mann-Whitney U-test if the data were nonparametric) for two group comparisons. Associations between categorical variables were analysed using the Chi-squared test. The interobserver agreement for the presence of pathological and imaging features was evaluated using unweighted $\kappa$ for binary variables, and weighted $\kappa$ for variables with an ordinal scale. The interobserver variation of the extent of the various abnormalities was evaluated using Spearman's rank correlation coefficient. Univariate and multivariate Cox proportional hazards regression models were used to identify the physiological and CT predictors of outcome. Patient survival between CT categories and radiology-pathology-concordant and -discordant groups was determined using the log-rank test and Kaplan-Meier method. A p-value $<0.05$ was considered to indicate a significant difference.

\section{Results}

Baseline characteristics and PFTs of the study subjects

The mean age of the 241 patients was $65.7 \pm 8.0$ years and $24 \%$ were female (table 1 ). The mean forced vital capacity (FVC \% predicted) was $63.8 \%$ and the mean diffusing capacity of the lung for carbon monoxide (DLCO \% pred) was $37.8 \%$. The mean interval between the CT scan and SLBx was 1.75 years (range 0.0110.4 years). When the maximal interval from CT scan to SLBx was limited to 1 year, 106 cases remained with a mean interval of 0.3 years (range 0.01-0.97 years). Baseline patient characteristics and physiological data for this smaller group of subjects are shown in online supplementary table S1, and are similar to those of the larger group.

\section{Interobserver agreement/correlation for CT findings and CT categorisation}

Pre-consensus interobserver agreement and correlations are shown in table 2. Interobserver agreement was excellent for the presence of reticular opacity and honeycombing $(\kappa=0.85-1.00)$, and substantial for the presence of diffuse mosaic attenuation/air-trapping, consolidation and emphysema $(\kappa=0.66-0.71)$. The interobserver agreement for CT categorisation was substantial $(\kappa=0.77$; table 3$)$.

\section{Comparison between radiological and pathological diagnoses}

The CT consensus diagnosis segregated according to pathological diagnosis is shown in table 4. Of those with UIP pattern on HRCT, 99 (97.1\%) had definite UIP or probable UIP patterns on biopsy (radiologypathology-concordant group) and 71 (94.7\%) of those with a pattern that was "inconsistent with UIP" had definite UIP or probable UIP on biopsy (radiology-pathology-discordant group; figure 1 and 2). Among the radiology-pathology-discordant cases, $51(71.8 \%)$ of the inconsistent CT findings were a result of

TABLE 1 Clinical and physiological differences among the computed tomography diagnostic categories

\begin{tabular}{|c|c|c|c|c|c|}
\hline & Total & UIP & Possible UIP & $\begin{array}{l}\text { Inconsistent } \\
\text { with UIP }\end{array}$ & p-value \\
\hline Subjects & 241 & 102 & 64 & 75 & \\
\hline Age years & $65.7 \pm 8.0$ & $67.4 \pm 7.5$ & $63.8 \pm 7.8^{*}$ & $65.0 \pm 8.5$ & 0.01 \\
\hline Duration of illness years & $2.0 \pm 2.1$ & $2.3 \pm 2.1$ & $1.7 \pm 1.7$ & $2.0 \pm 2.3$ & 0.236 \\
\hline Smoking ever/never & $175 / 66$ & $82 / 20$ & $42 / 22$ & $51 / 24$ & 0.065 \\
\hline 6-min walking distance $\mathrm{m}$ & $326.9 \pm 136.9$ & $320.3 \pm 157.7$ & $356.40 \pm 117.4$ & $310.9 \pm 118.2$ & 0.124 \\
\hline DLco \% pred & $37.8 \pm 13.4$ & $35.3 \pm 13.6$ & $41.7 \pm 14.8^{*}$ & $38.0 \pm 11.9$ & 0.01 \\
\hline FEV $1 /$ FVC $\%$ & $77.6 \pm 1.9$ & $77.3 \pm 1.6$ & $77.9 \pm 1.6$ & $77.9 \pm 2.3$ & 0.029 \\
\hline FVC \% pred & $63.8 \pm 16.3$ & $64.8 \pm 16.0$ & $65.6 \pm 17.5$ & $61.0 \pm 15.3$ & 0.192 \\
\hline Total lung capacity L & $4.0 \pm 1.1$ & $4.1 \pm 1.1$ & $4.2 \pm 1.2$ & $3.7 \pm 0.9 * .7$ & 0.011 \\
\hline History of antigen exposure & 13 (5.39) & $6(5.88)$ & $4(6.25)$ & $3(4.00)$ & 0.491 \\
\hline
\end{tabular}

Data are presented as $n$, mean \pm SD or $n(\%)$, unless otherwise stated. UIP: usual interstitial pneumonia; DLco: diffusing capacity of the lung for carbon monoxide; FEV1: forced expiratory volume in $1 \mathrm{~s}$; FVC: forced vital capacity. ${ }^{\#}$ : higher score indicates worse function. ${ }^{*}$ : $p<0.05$ versus UIP group; " : $p<0.05$ versus possible UIP group. 
TABLE 2 Extent of computed tomography findings and pre-consensus interobserver agreement/correlation

\begin{tabular}{|c|c|c|c|}
\hline & \multirow[t]{2}{*}{ Number of cases or extent } & \multicolumn{2}{|c|}{$\begin{array}{c}\text { Pre-consensus } \\
\text { interobserver } \\
\text { agreement/correlation }\end{array}$} \\
\hline & & $\kappa$ or $\rho$-value & p-value \\
\hline Reticular abnormality & $241(100.0)$ & $0.99^{\pi}$ & $<0.001$ \\
\hline Extent of reticular abnormality $\#$ & $30.1 \pm 8.7$ & $0.15^{+}$ & 0.008 \\
\hline Honeycombing & $131(54.4)$ & $0.85^{\natural}$ & $<0.001$ \\
\hline Extent of honeycombing ${ }^{\#}$ & $9.1 \pm 11.0$ & $0.65^{+}$ & $<0.001$ \\
\hline Extensive ground-glass abnormality & $21(8.7)$ & $0.20^{9}$ & $<0.001$ \\
\hline Profuse micronodules & $13(5.4)$ & $0.55^{\natural}$ & $<0.001$ \\
\hline Discrete cysts & $0(0)$ & 0.99 १ & $<0.001$ \\
\hline Diffuse mosaic attenuation/air trapping & $52(21.6)$ & $0.71^{\uparrow}$ & $<0.001$ \\
\hline Consolidation & $3(1.2)$ & $0.66^{\text {शा }}$ & $<0.001$ \\
\hline Emphysema & 48 (19.9) & $0.71^{\Uparrow}$ & $<0.001$ \\
\hline Craniocaudal distribution & & $0.4^{9}$ & $<0.001$ \\
\hline Lower lung predominance & $182(75.5)$ & $0.51^{\text {ๆ }}$ & $<0.001$ \\
\hline Upper or mid-lung predominance & $20(8.3)$ & $0.31^{\Uparrow}$ & $<0.001$ \\
\hline Diffuse distribution & $39(16.2)$ & 0.29 9 & $<0.001$ \\
\hline Axial distribution & & $0.34^{\text {व }}$ & $<0.001$ \\
\hline Peripheral predominance & 208 (86.3) & 0.39 9 & $<0.001$ \\
\hline Central/PBV predominance & $12(5.0)$ & $0.30^{9}$ & $<0.001$ \\
\hline Diffuse distribution & $21(8.7)$ & $0.29^{9}$ & $<0.001$ \\
\hline
\end{tabular}

Data are presented as $n(\%)$ or mean \pm SD, unless otherwise stated. PBV: peribronchovascular. ${ }^{\#}$ : percentage of lung parenchyma; ${ }^{\uparrow}: \kappa$ analysis; ${ }^{+}: \rho$-value with Spearman's rank correlation.

diffuse mosaic/air trapping, 16 (22.5\%) were a result of extensive ground-glass abnormalities, 17 (23.9\%) were a result of the predominance of signs in the upper or mid-zones of the lungs, 28 (39.4\%) were a result of diffuse craniocaudal distribution, nine (12.7\%) were a result of a peribronchovascular predominance and $19(26.8 \%)$ were a result of diffuse axial distribution. Several subjects had more than one inconsistent CT finding. The results were similar when the analysis was restricted to the 106 individuals who underwent SLBx within 1 year of their CT scan (online supplementary tables S2 and S3).

\section{Clinical and physiological differences among CT diagnoses}

The clinical and physiological differences between the CT diagnostic categories are shown in table 1 . The patients with a definite HRCT pattern of UIP were older and had a lower DLCO \% pred, compared to those with a possible UIP pattern. In addition, they had a higher total lung capacity than those with a pattern that was inconsistent with UIP.

In terms of the $\mathrm{CT}$ findings within the pathological diagnostic categories, there were no significant differences in CT findings between the pathological diagnoses (table 5).

Physiological differences between radiology-pathology-concordant and -discordant groups

The individuals in the radiology-pathology-concordant group tended to be older $(\mathrm{p}=0.036)$, more likely to be current or former smokers $(\mathrm{p}=0.049)$, had lower forced expiratory volume in $1 \mathrm{~s}$ (FEV1)/FVC

TABLE 3 Interobserver agreement for computed tomography categorisation

\begin{tabular}{lccc} 
Observer 2 & \multicolumn{3}{c}{ Observer 1 } \\
\cline { 2 - 4 } & UIP & Possible UIP & Inconsistent with UIP \\
\hline UIP & 85 & 11 & 7 \\
Possible UIP & 1 & 53 & 11 \\
Inconsistent with UIP & 4 & 2 & 67 \\
\hline
\end{tabular}

Data are presented as n. UIP: usual interstitial pneumonia. 
TABLE 4 Computed tomography consensus diagnosis segregated by the pathological diagnosis

\begin{tabular}{lccccc} 
& \multicolumn{2}{c}{ Pathological diagnosis } & \multirow{2}{*}{ Total } \\
\cline { 2 - 4 } & Definite UIP & Probable UIP & Possible UIP & Not UIP & \\
\hline UIP & 82 & 17 & 1 & 2 & 102 \\
Possible UIP & 51 & 9 & 4 & 0 & 64 \\
Inconsistent with UIP & 55 & 16 & 4 & 0 & 75 \\
Total & 188 & 42 & 9 & 2 & 241 \\
\hline
\end{tabular}

Data are presented as n. UIP: usual interstitial pneumonia.

$(\mathrm{p}=0.032)$ and higher total lung capacity $(\mathrm{p}=0.01)$ than those in the radiology-pathology-discordant group. There were no significant differences between groups in terms of the duration of illness, DLCO \% pred, history of antigen exposure or history of gastroesophageal reflux (table 6). When the analysis was restricted to the 106 individuals who underwent a SLBx within 1 year of the CT scan, the findings were similar (online supplementary table S4).

\section{Detailed pathological evaluation between concordant and discordant groups}

To assess the features that might correlate with the radiological findings that led to an "inconsistent" CT interpretation, we performed a detailed histological re-review of 100 cases (31 randomly selected radiology-pathology-concordant cases and 69 radiology-pathology-discordant cases). No significant differences were found in terms of the presence and degree of peribronchiolar metaplasia, chronic inflammation and lymphoid hyperplasia, giant cells, granulomas, bridging fibrosis, organising pneumonia/ acute lung injury or airway-centered interstitial changes (online supplementary table S5). 12 radiologypathology-concordant cases and 37 radiology-pathology-discordant cases had an interval of $<1$ year between their CT and lung biopsy, and, again, no significant differences were found between the concordant and discordant cases (online supplementary table S6). Interobserver agreement among the two pathologists who performed the image re-review were as follows: peribronchiolar metaplasia $\kappa=0.34$; chronic inflammation and lymphoid hyperplasia $\kappa=0.28$; giant cells $\kappa=0.29$; granuloma $\kappa=-0.04$; bridging fibrosis $\kappa=0.02$; organising pneumonia/acute lung injury $\kappa=0.41$; and airway-centered interstitial changes $\kappa=0.16$.

\section{Survival}

The median duration of follow-up for subjects included in this study was 47.5 weeks. 38 patients died during the follow-up period. The results of the univariate Cox regression analysis regarding the relationship between survival and physiological or CT scan features are shown in online supplementary table S7. In the univariate analysis, 6MWT, total score on SGRQ, post-walk Borg dyspnoea score, FVC \% pred and DLCO \% pred were significant predictors of survival (HR 0.99, 1.08, 1.36, 0.94 and 0.89, respectively). In the multivariate analysis, the total score on the SGRQ was the only significant predictor of mortality (HR 1.059). Out of the 99 radiology-pathology-concordant subjects, eight (8.1\%) patients died, and three $(4.2 \%)$ out of the 71 patients in the radiology-pathology-discordant group died $(\mathrm{p}=0.314)$. The median survival of patients with radiology-pathology-concordance and -discordance was 38.6 weeks and 51.7 weeks, respectively. The survival of the radiology-pathology-concordant group did not differ significantly from that of the radiology-pathology-discordant group (log-rank test $\mathrm{p}=0.334$ ) during the follow-up period.

\section{Discussion}

In this study of subjects enrolled in IPF clinical trials, $94.7 \%$ of those with a pattern on HRCT that was inconsistent with UIP also showed definite or probable UIP on histological evaluation. We did not identify any difference in the pathological findings observed between the patients in the radiologypathology-concordant and -discordant groups. However, patients in the concordant group were older, had a greater smoking history, larger lung volumes and a slightly lower FEV1/FVC ratio than those in the discordant group. The differences in PFTs could be related to the higher proportion of smokers in the concordant group. Although the numbers were low, there was no significant difference in survival between these groups.

In this study, a CT diagnosis of UIP was made in 43.0\% (99 out of 230) of patients with definite or probable UIP. This relatively low sensitivity is similar to the findings of previous reports $[2,4,9,10]$. In addition, $55(29.3 \%)$ out of 188 patients with a definite pathological UIP pattern had a HRCT pattern that 

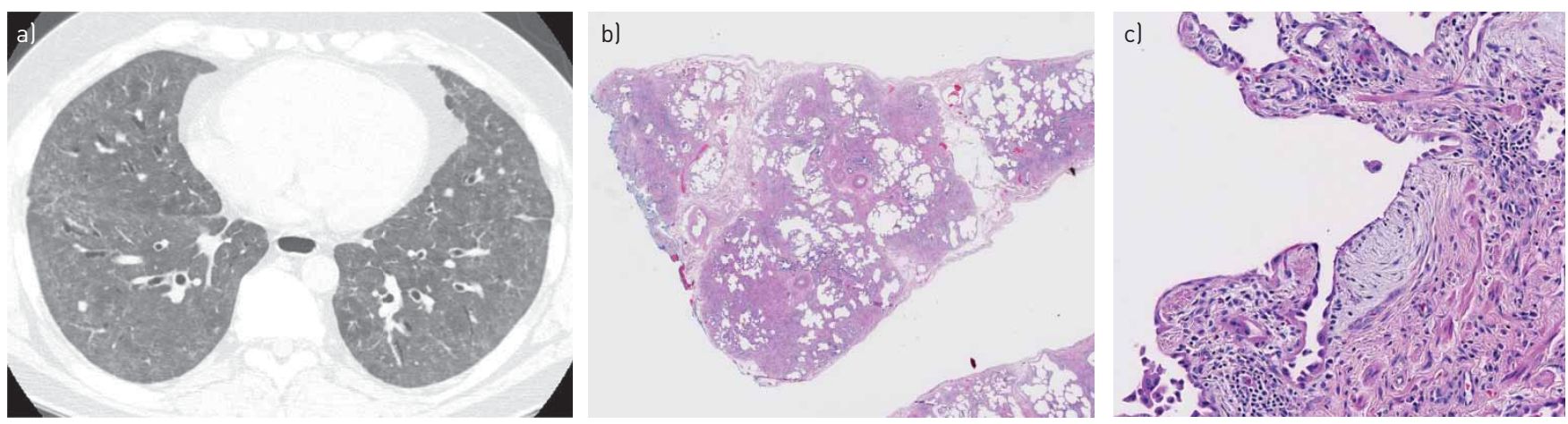

FIGURE 1 a) High-resolution computed tomography (HRCT) pattern inconsistent with usual interstitial pneumonia (UIP). A surgical lung biopsy was obtained from this patient 87 days prior to computed tomography. Axial HRCT demonstrates an extensive ground-glass abnormality. b) Surgical lung biopsy specimen demonstrating a pattern of UIP; scarring and honeycombing are demonstrated. Scanning power. c) A fibroblast focus is present (haematoxylin and eosin). Intermediate power.

was inconsistent with UIP, which is in accordance with previous reports [9-11]. There was inevitable selection bias in our population: patients with a UIP pattern on HRCT do not usually undergo SLBx. However, in our cohort, 102 (42.3\%) out of 241 patients had typical UIP pattern (table 2). With more cases of typical HRCT pattern included, the rates of concordant diagnosis between HRCT and pathology would increase. Likewise, the indications for SLBx would be different among various institutions included in this study. In addition, all subjects in this study were preselected and diagnosed with IPF at academic centres, and approved for entry into the clinical trials. Thus, the findings are not generalisable to the
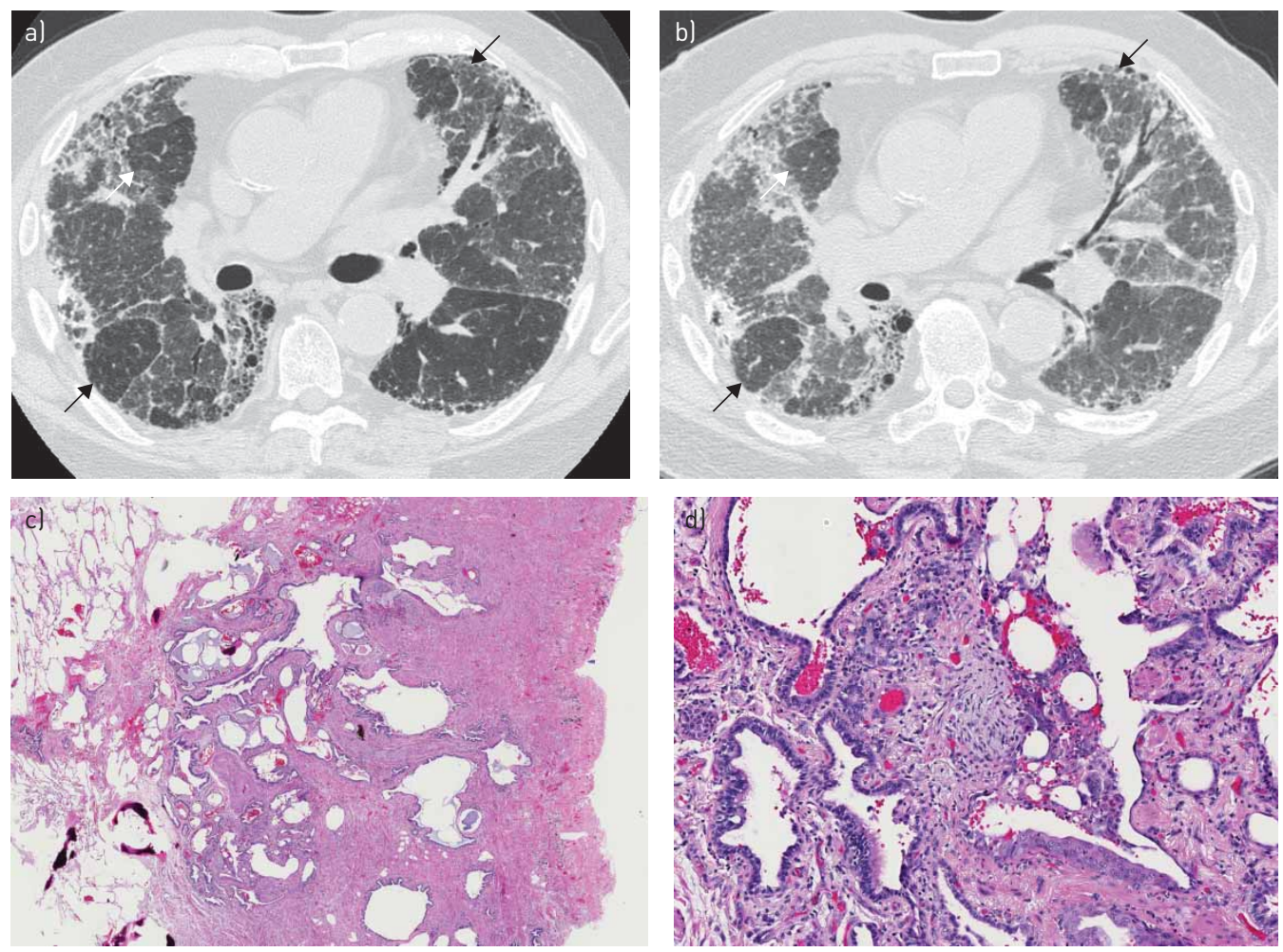

FIGURE 2 a, b) Imaging pattern inconsistent with usual interstitial pneumonia (UIP). A surgical lung biopsy was obtained 88 days prior to computed tomography (CT). a) Axial high-resolution computed tomography (HRCT) during inspiration shows mosaic attenuation involving three lobes (arrows). b) Axial HRCT during expiration demonstrates air trapping in corresponding areas (arrows). Air trapping should be present in at least three lobes for the CT pattern to be considered as inconsistent with UIP. c) Surgical lung biopsy specimen demonstrating a typical UIP pattern; scarring and honeycombing are demonstrated. Scanning power. d) A fibroblast focus is present (haematoxylin and eosin). Intermediate power. 
TABLE 5 Computed tomography findings among pathological usual interstitial pneumonia (UIP) categories

\begin{tabular}{|c|c|c|c|c|c|}
\hline & \multicolumn{4}{|c|}{ Pathological diagnosis } & \multirow[t]{2}{*}{ p-value } \\
\hline & Definite UIP & Probable UIP & Possible UIP & Not UIP & \\
\hline Subjects & 188 & 42 & 9 & 2 & \\
\hline Extent of reticular abnormality ${ }^{\#}$ & $30.2 \pm 8.7$ & $30.2 \pm 8.8$ & $29.4 \pm 9.2$ & $22.5 \pm 3.5$ & 0.512 \\
\hline Honeycombing & $103(54.8)$ & $23(54.8)$ & 3 (33.3) & $2(100)$ & 0.347 \\
\hline Extent of honeycombing ${ }^{\#}$ & $15.8 \pm 9.0$ & $16.9 \pm 14.1$ & $13.8 \pm 5.9$ & $20.0 \pm 7.1$ & 0.255 \\
\hline Discrete cysts & $0(0)$ & $0(0)$ & $0(0)$ & $0(0)$ & NA \\
\hline Diffuse mosaic attenuation/air trapping & $40(21.3)$ & $11(26.2)$ & $1(11.1)$ & $0(0)$ & 0.643 \\
\hline Consolidation & $2(1.1)$ & $0(0)$ & $1(11.1)$ & $0(0)$ & 0.052 \\
\hline Emphysema & $33(17.6)$ & $13(31)$ & $2(22.2)$ & $0(0)$ & 0.222 \\
\hline Craniocaudal distribution & & & & & 0.993 \\
\hline Lower lung predominance & $141(75)$ & $32(76.2)$ & $7(77.8)$ & $2(100)$ & \\
\hline Central/PBV predominance & 7 (3.7) & $4(9.5)$ & $1(11.1)$ & $0(0)$ & \\
\hline Diffuse distribution & $16(8.5)$ & 5 (11.9) & $0(0)$ & $0(0)$ & \\
\hline
\end{tabular}

Data are presented as $\mathrm{n}, \mathrm{n}(\%)$ or mean $\pm \mathrm{SD}$, unless otherwise stated. PBV: peribronchovascular; NA: not answered. \#: percentage of lung parenchyma.

broader population of subjects with fibrosing interstitial pneumonia, particularly given the lack of a "control group" of patients with CT findings inconsistent with UIP and biopsy also inconsistent with UIP.

Diffuse mosaic attenuation/air trapping was observed in $21.3 \%$ (40 out of 188) patients with a definite pathological UIP pattern. This feature was also identified with a higher prevalence in a previous study, which identified air trapping in 35\% of patients with UIP [12]. However, in our study, a higher threshold was applied for mosaic attenuation/air trapping, as these findings were required to be present bilaterally or in three or more lobes. Although air trapping is associated with chronic hypersensitivity pneumonitis [12], we did not identify any differences in reported history of antigen exposure between the radiologypathology-concordant and -discordant groups, and careful histological re-review showed no evidence for hypersensitivity pneumonitis. It is still possible that some of the discordant cases may have represented

TABLE 6 Clinical and physiological differences between concordant and discordant cases

\begin{tabular}{|c|c|c|c|}
\hline & Concordant cases & Discordant cases & p-value \\
\hline Subjects & 99 & 71 & \\
\hline Sex male/female & $78 / 21$ & $48 / 23$ & 0.101 \\
\hline Age years & $67.5 \pm 7.7$ & $64.9 \pm 8.7$ & $0.036^{\text {1 }}$ \\
\hline Duration of illness years & $2.2 \pm 2.1$ & $1.9 \pm 2.1$ & 0.307 \\
\hline Smokers ever/never & $80 / 19$ & $48 / 23$ & $0.049^{\text {9 }}$ \\
\hline Post-walk Borg dyspnoea score ${ }^{\#}$ & $2.92 \pm 1.82$ & $2.91 \pm 1.84$ & 0.99 \\
\hline St George's Respiratory Questionnaire total score \# & $46.6 \pm 16.7$ & $48.3 \pm 18.1$ & 0.53 \\
\hline 6-min walking distance $\mathrm{m}$ & $320.0 \pm 157.3$ & $308.6 \pm 12.0$ & 0.612 \\
\hline DLco \% pred & $35.0 \pm 13.3$ & $38.2 \pm 12.2$ & 0.114 \\
\hline FEV1/FVC \% & $77.2 \pm 1.6$ & $77.9 \pm 2.4$ & $0.032^{\pi}$ \\
\hline FVC $\%$ pred & $64.6 \pm 16.2$ & $61.4 \pm 15.5$ & 0.208 \\
\hline Total lung capacity L & $4.1 \pm 1.1$ & $3.7 \pm 0.9$ & $0.010^{1}$ \\
\hline History of antigen exposure & $6(6.1)$ & $3(4.1)$ & 0.598 \\
\hline Gastro-oesophageal reflux symptoms & $50(70.4)$ & $62(62.6)$ & 0.371 \\
\hline \multicolumn{4}{|c|}{$\begin{array}{l}\text { Data are presented as } \mathrm{n} \text {, mean } \pm \text { SD or } \mathrm{n}(\%) \text {, unless otherwise stated. DLCo: diffusing capacity of the lung for } \\
\text { carbon monoxide; FEV1: forced expiratory volume in } 1 \mathrm{~s} \text {; FVC: forced vital capacity. \#: higher score indicates } \\
\text { poorer function; }{ }^{\circ} \text { : statistically significant difference between discordant and concordant cases (p<0.05). }\end{array}$} \\
\hline
\end{tabular}


hypersensitivity pneumonitis, as $20-40 \%$ of cases of chronic hypersensitivity pneumonitis may not have a discoverable antigen $[13,14]$. However, we must conclude that most subjects in our study with mosaic attenuation and air trapping actually had IPF. Current guidelines suggesting that these CT features are inconsistent with the diagnosis of IPF are probaby too restrictive. However, detailed environmental evaluation and SLBx is probably necessary in such patients to exclude the possibility of chronic hypersensitivity pneumonitis. This need is emphasised by a recent prospective study which showed that $>40 \%$ of patients presenting with features of IPF had chronic hypersensitivity pneumonitis [15].

In our study, 60 (94\%) out of 64 patients with possible UIP on HRCT had histologically definite or probable UIP. Similarly, in a different population, 79 (94\%) out of 84 subjects enrolled in a clinical trial who had possible UIP on HRCT showed pathologically definite or probable UIP [10]. A different study categorised biopsy-confirmed UIP into four CT patterns: classic UIP, fibrosis without honeycombing $(\mathrm{FnoH})$, minimal fibrosis and ground-glass present [16], and concluded that the $\mathrm{FnoH}$ pattern may be diagnostic of clinical UIP. A third recent study showed that $82 \%$ of subjects with "probable UIP" on CT (typical findings of UIP without honeycombing) had histological UIP [17]. Our study (albeit from a highly selected population) supports the suggestion from these studies that the findings of possible UIP on HRCT may be diagnostic of UIP, and SLBx may not be necessary in these cases to confirm or clarify the diagnosis of IPF.

In our study, there was no significant difference in outcomes between the radiology-pathology-concordant and-discordant groups. This is similar to some previous reports [4, 9]. However, other studies found a significant decrease in survival rates in the group with radiologically and pathologically concordant UIP [2, 5]. These differences may be due to differing population selection, in addition to the relatively small number of deaths and relatively short duration of follow-up in our study.

In addition to the selection bias discussed earlier and the retrospective nature of the study, a further limitation is the lengthy mean interval between the SLBx and CT scan (1.47 years), reflecting the relatively long interval between diagnosis and study enrolment. It is certainly possible that the CT pattern might have changed in the interval after the biopsy. However, the CT pattern would be expected to have become more typical of UIP as the disease progressed. Also, we found similar results when the biopsy-CT interval was restricted to $\leqslant 1$ year. There was also a relatively large observer variation for several of the HRCT and histological features, which may indicate a subjective bias in identifying these features. Some of this variation, particularly for the histological variables, could be due to the tendency of the $\kappa$-value to be reduced when the proportion of normal or abnormal findings is low [18].

In conclusion, in this selected population, $94.7 \%$ of those with a pattern that was inconsistent with UIP on HRCT showed definite or probable UIP on histological evaluation. There were no major pathological differences between these radiology-pathology-concordant and -discordant groups. This indicates that subjects with CT findings "inconsistent with UIP" may have IPF, and suggests that this term is misleading.

\section{References}

1 Travis WD, Costabel U, Hansell DM, et al. An official American Thoracic Society/European Respiratory Society statement: update of the international multidisciplinary classification of the idiopathic interstitial pneumonias. Am J Respir Crit Care Med 2013; 188: 733-748.

2 Flaherty KR, Thwaite EL, Kazerooni EA, et al. Radiological versus histological diagnosis in UIP and NSIP: survival implications. Thorax 2003; 58: 143-148.

3 Raghu G, Collard HR, Egan JJ, et al. An official ATS/ERS/JRS/ALAT statement: idiopathic pulmonary fibrosis: evidence-based guidelines for diagnosis and management. Am J Respir Crit Care Med 2011; 183: 788-824.

4 Sverzellati N, Wells AU, Tomassetti S, et al. Biopsy-proved idiopathic pulmonary fibrosis: spectrum of nondiagnostic thin-section CT diagnoses. Radiology 2010; 254: 957-964.

5 Sumikawa H, Johkoh T, Fujimoto K, et al. Pathologically proved nonspecific interstitial pneumonia: CT pattern analysis as compared with usual interstitial pneumonia CT pattern. Radiology 2014; 272: 549-556.

6 Idiopathic Pulmonary Fibrosis Clinical Research Network, Raghu G, Anstrom KJ, et al. Prednisone, azathioprine, and $N$-acetylcysteine for pulmonary fibrosis. N Engl J Med 2012; 366: 1968-1977.

7 Idiopathic Pulmonary Fibrosis Clinical Research Network, Zisman DA, Schwarz M, et al. A controlled trial of sildenafil in advanced idiopathic pulmonary fibrosis. N Engl J Med 2010; 363: 620-628.

8 Noth I, Anstrom KJ, Calvert SB, et al. A placebo-controlled randomized trial of warfarin in idiopathic pulmonary fibrosis. Am J Respir Crit Care Med 2012; 186: 88-95.

9 Sumikawa H, Johkoh T, Colby TV, et al. Computed tomography findings in pathological usual interstitial pneumonia: relationship to survival. Am J Respir Crit Care Med 2008; 177: 433-439.

10 Raghu G, Lynch D, Godwin JD, et al. Diagnosis of idiopathic pulmonary fibrosis with high-resolution CT in patients with little or no radiological evidence of honeycombing: secondary analysis of a randomised, controlled trial. Lancet Respir Med 2014; 2: 277-284.

11 Sumikawa $\mathrm{H}$, Johkoh $\mathrm{T}$, Ichikado $\mathrm{K}$, et al. Usual interstitial pneumonia and chronic idiopathic interstitial pneumonia: analysis of CT appearance in 92 patients. Radiology 2006; 241: 258-266.

12 Silva CI, Müller NL, Lynch DA, et al. Chronic hypersensitivity pneumonitis: differentiation from idiopathic pulmonary fibrosis and nonspecific interstitial pneumonia by using thin-section CT. Radiology 2008; 246 : 288-297. 
13 Selman M, Pardo A, King TE Jr. Hypersensitivity pneumonitis: insights in diagnosis and pathobiology. Am J Respir Crit Care Med 2012; 186: 314-324.

14 Costabel U, Bonella F, Guzman J. Chronic hypersensitivity pneumonitis. Clin Chest Med 2012; 33: 151-163.

15 Morell F, Villar A, Montero MA, et al. Chronic hypersensitivity pneumonitis in patients diagnosed with idiopathic pulmonary fibrosis: a prospective case-cohort study. Lancet Respir Med 2013; 1: 685-694.

16 Gruden JF, Panse PM, Leslie KO, et al. UIP diagnosed at surgical lung biopsy, 2000-2009: HRCT patterns and proposed classification system. AJR Am J Roentgenol 2013; 200: W458-W467.

17 Chung JH, Chawla A, Peljto AL, et al. CT scan findings of probable usual interstitial pneumonitis have a high predictive value for histologic usual interstitial pneumonitis. Chest 2015; 147: 450-459.

18 Feinstein AR, Cicchetti DV. High agreement but low kappa: I. The problems of two paradoxes. J Clin Epidemiol 1990; 43: 543-549. 